\title{
Right Ventricular Perforation after Pacemaker Implantation in an Elderly Woman: A Case Report
}

\author{
Fernando S. Montenegro \\ Adventist Silvestre Hospital, Guararapes Street 274 , Cosme Velho, Rio de Janeiro 22241220, Brasil
}

\begin{abstract}
Background: Cardiac pacing is a proven and effective treatment used worldwide with a low incidence of complications depending on the patient group. Late lead perforation is a rare complication without a standard treatment. Case summary: We report a case of a 98-year old woman submitted to pacemaker implantation due to symptomatic second-degree atrio-ventricular block. After two days she returned to the hospital with thoracic pain and was diagnosed with RV (right ventricular) lead perforation. She was submitted to cardiac surgery with lead extraction and RV repair. Discussion: There are two major types of complications after pacemaker implantation, mechanical and infection. Reported perforation rates after pacemaker implantation range from $0.1 \%-0.8 \%$. While acute lead perforation is very well described, late lead perforation is only described in case reports with a low rate of tamponade or death. Management of subacute or delayed RV lead perforation is not well defined due to very limited data. Question remains on whether to extract the lead or not.
\end{abstract}

Key words: Pacemaker, cardiac surgery, arrhythmia, case report.

\section{Introduction}

Cardiac pacing is a proven and effective treatment in the management of many cardiac arrhythmias. Population aging and technical advances have led to a substantial increase in device implantation. The rate of acute complications from device implantation ranges from $3 \%$ to $7 \%$ depending on the patient group [1].

The extreme elderly (age $\geq 80$ years) are the most rapidly growing segment of population worldwide and pacemakers are commonly implanted in this population [2]. There are few reports of pacemaker implant complications and outcomes in the extreme elderly and there is a persistent exclusion of this population from ongoing clinical trials. This paucity of data makes it difficult to estimate risks of device implantation and to determine the best management of such complications [3].

\section{Case Report}

DPN, a 97-year-old woman, is admitted to the

Corresponding author: Fernando S Montenegro, M.D., MSc, research fields: coronary artery disease and cardiac surgery. hospital for permanent pacemaker implant due to symptomatic second-degree atrio-ventricular block (DDDR mode, lower rate set at 60 beats per minute). She had a history of hypertension in use of valsartan. No perioperative complications were reported. Chest X-ray next day after the procedure showed RV (right ventricular) pacemaker lead overlying the left heart margin (Fig. 1). Thorax CT (computed tomography) scan did not show signs of perforation with both leads in the expected position. She was discharged from the hospital the next day. After two days she returned to the emergency department with palpitations and thoracic pain. Her blood pressure was $132 / 53 \mathrm{mmHg}$ with regular heart rate and rhythm at $67 \mathrm{bpm}$ and oxygen saturation of $98 \%$ on room air. Cardiovascular exam revealed no murmurs, gallops, jugular venous distention, or lower limb edema. Lung auscultation revealed slightly diminished breath sounds at left base. Device interrogation revealed no capture in the RV lead with diaphragmatic stimulation in higher outputs and episodes of atrial fibrillation. Chest X-ray showed a mild left-sided pleural effusion, which was not present in the previous exam (Fig. 2). Transthoracic 


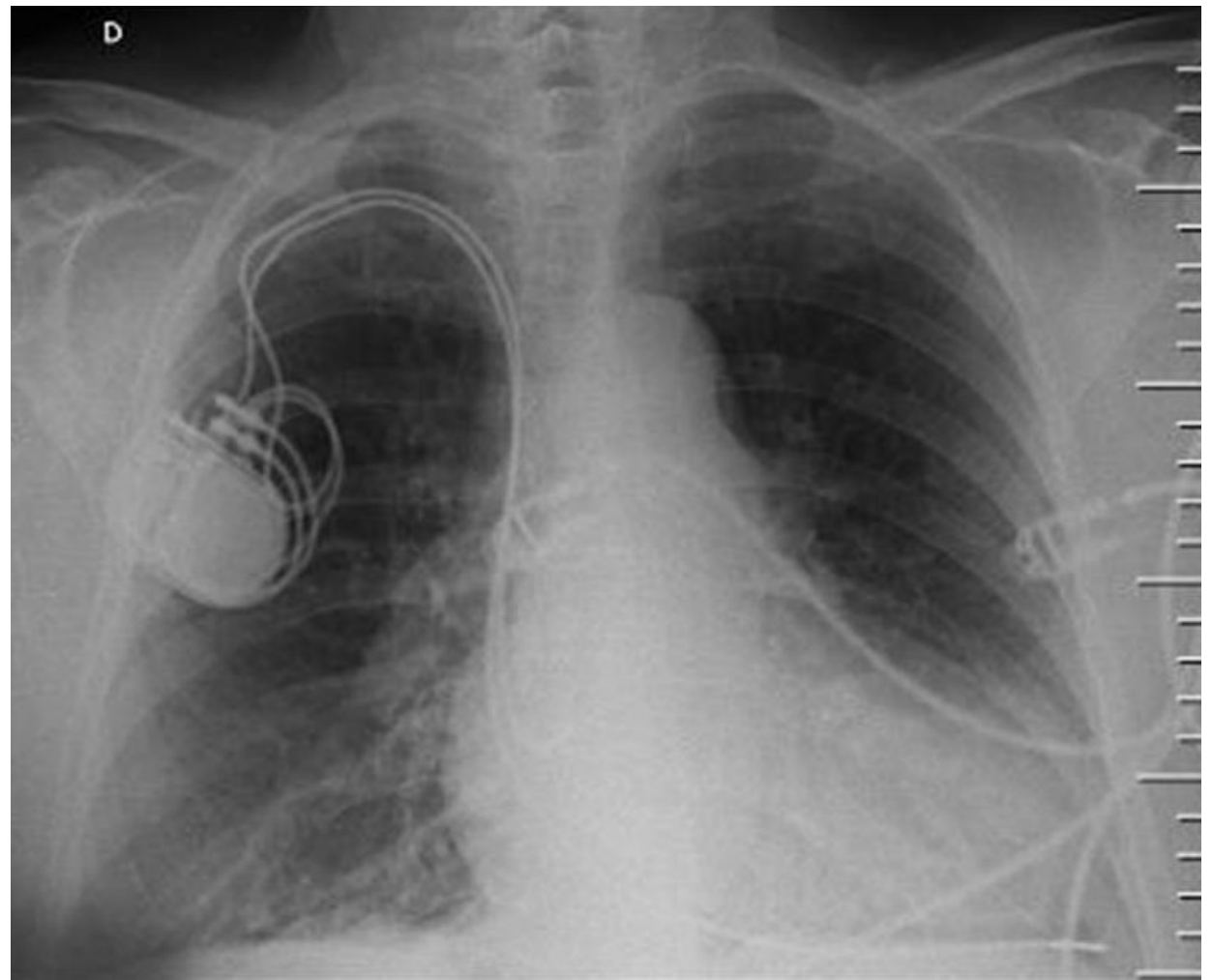

Fig. 1 Chest X-ray in the morning after pacemaker implantation.

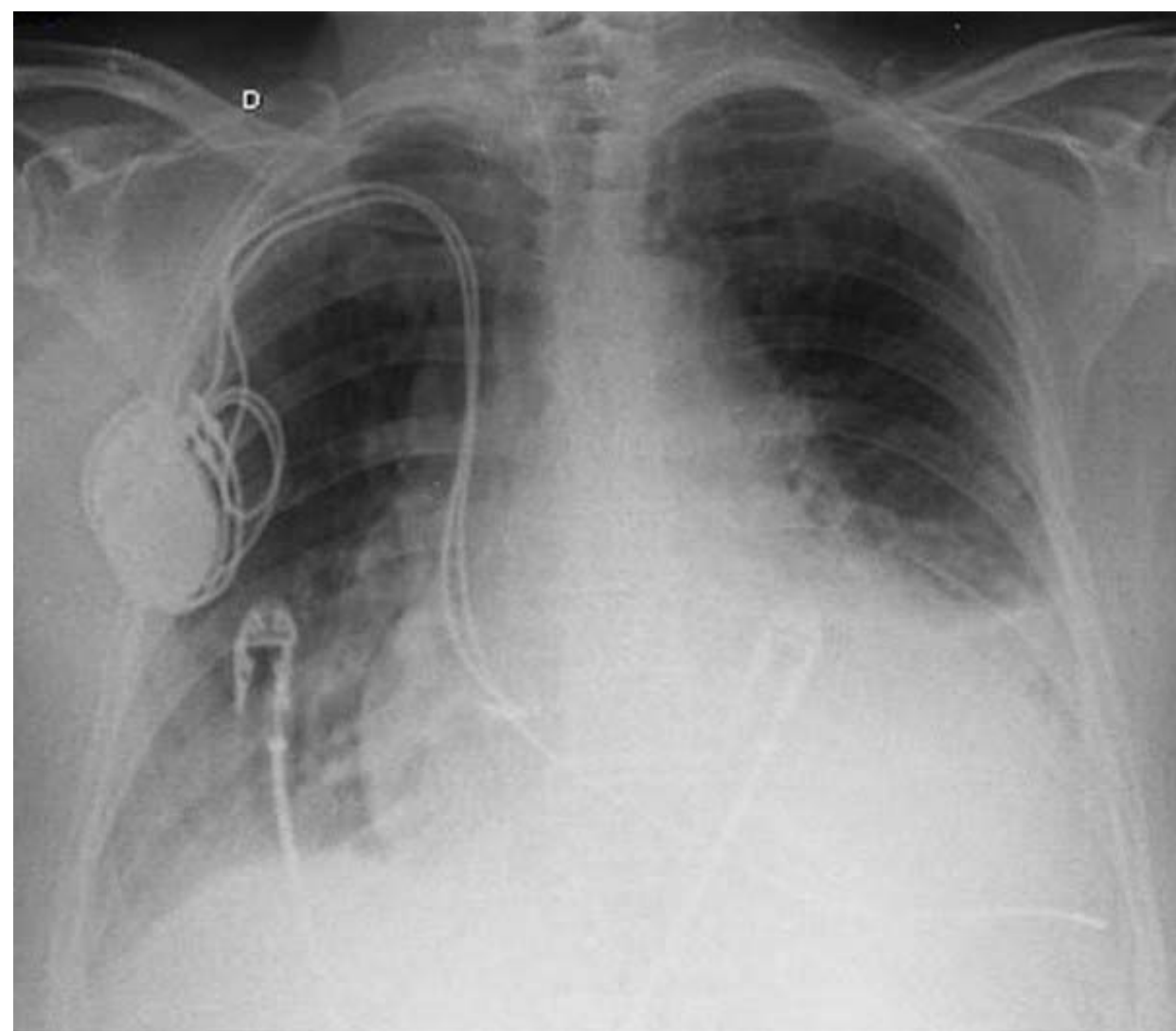

Fig. 2 Chest X-ray six days after pacemaker implantation.

A mild left-sided pleural effusion can be observed which was not presented in the chest X-ray after de procedure. 


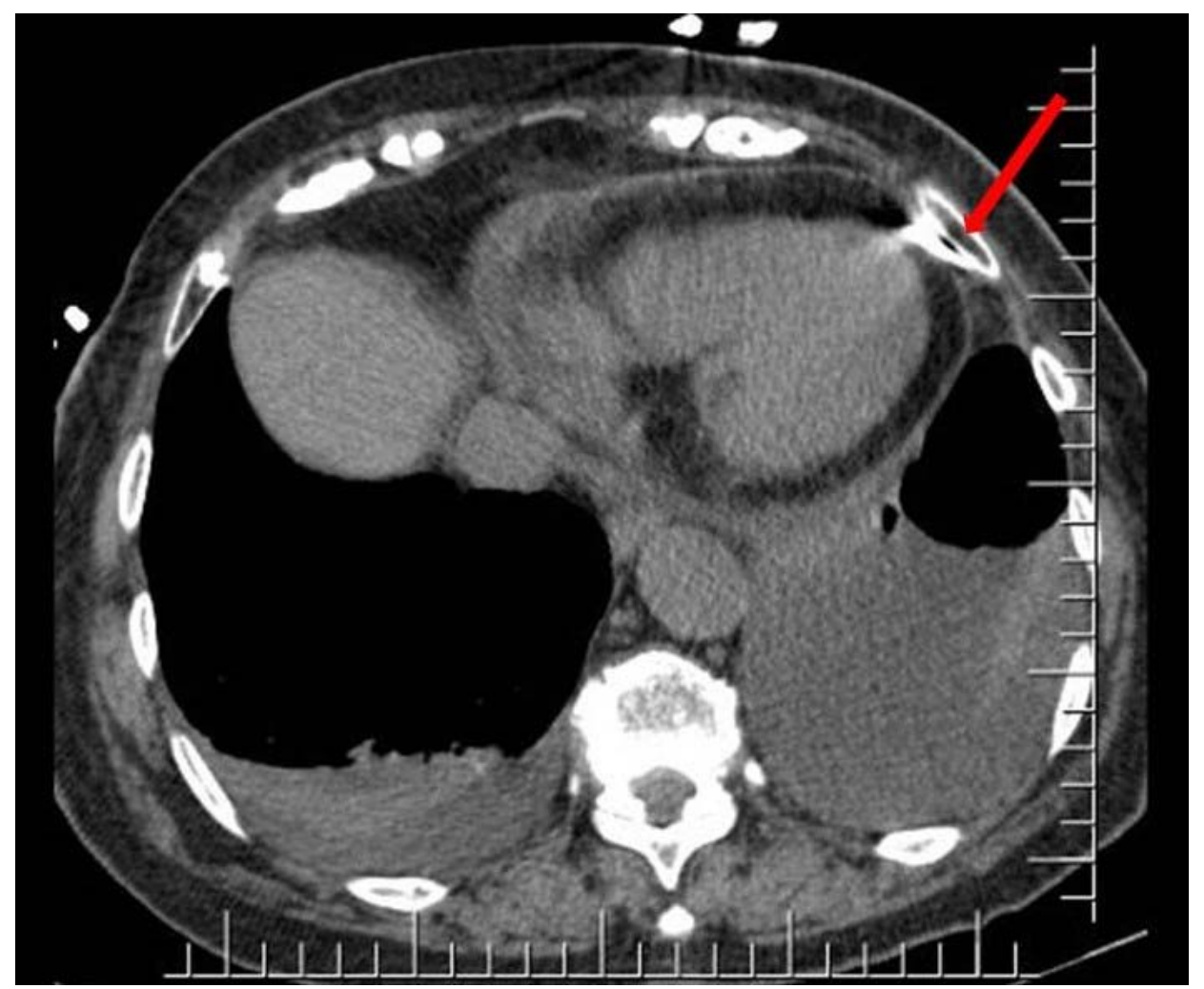

Fig. 3 CT scan six days after pacemaker implantation revealed the tip of the RV lead penetrating the anterior wall of the right ventricle (red arrow) with moderate left pleural effusion and a small-localized pericardial effusion.

echocardiogram showed a normal left ventricular systolic function with an ejection fraction of $78 \%$ with a small collection in the pericardial sac and no evidence of tamponade, and the both leads visualized in the right atria and ventricle. A new thorax CT scan revealed the tip of the RV lead penetrating the anterior wall of the right ventricle and terminating in the left anterior chest with moderate left pleural effusion and a small-localized pericardial effusion (Fig. 3). The patient was taken to the operating room in the same day where she underwent a median sternotomy. The pacemaker lead was found to be protruding out of the right ventricle and was removed. The perforation of the RV wall was repaired. The hemothorax and hemopericardium were evacuated. An epicardial lead was placed. In the postoperative period, she presented with acute renal failure needing dialysis therapy, nosocomial infection and paroxysmal episodes of atrial fibrillation. She was discharged home 30 days after admission without dialysis therapy. She was reassessed in the ambulatory 5 months after discharge without any cardiovascular complaints.

\section{Discussion}

There are two major types of complications after pacemaker implantation, mechanical, including pneumothorax, myocardial perforation and lead malposition and infection. Reported perforation rates after pacemaker implantation range from $0.1 \%-0.8 \%$. Perforations occurring within $24 \mathrm{~h}$ after implantation are labeled as acute; those occurring within one month after implantation are subacute, while perforations occurring after one month are labeled as chronic [4]. Pacemaker perforations may occur through the walls of the large veins, atria, or ventricles. Perforations involving the RV apex, which is thinner, are more commonly than the intraventricular septum or the outflow tract [5]. The most common symptom is thoracic pain followed by diaphragmatic stimulation. Nevertheless, up to one quarter of patients with perforations are asymptomatic [6].

While acute lead perforation is very well described, 
causing in rare cases cardiac tamponade and death, late lead perforation is only described in case reports with a low rate of tamponade or death. Right heart is a low-pressure system and perforation may be sealed by a combination of the lead itself, muscle contraction, and perhaps fibrosis, resulting in no sequel [4].

Management of subacute or delayed RV lead perforation is not well defined due to very limited data. Question remains on whether to extract the lead or not and if so, whether to extract perforating leads percutaneously or surgically.

Percutaneous extraction has been demonstrated to be a safe procedure with low rates of complications. Labordiere et al. [7] in series of eleven consecutive patients with subacute or delayed perforation, removed leads by simple traction under fluoroscopic guidance in the operating room, with surgical backup support with only the occurrence in 1 patient of tamponade requiring percutaneous pericardiocentesis and urgent surgical revision. However, in this series the mean age was 62 years old, much younger than our patient.

Milgliore et al. [8] found similar results in 14 consecutive patients with mean age of 71 years (oldest patient was 83 years old) whose leads were successfully extracted by direct manual traction in the absence of any complications.

However, there are no data regarding safety of lead extraction on an older population (more than 90 years old). In this population managing this complication should take into account clinical status, life expectancy and quality of life.

\section{Conclusions}

To the best of our knowledge, this is the first case report describing subacute lead perforation in a ninety eight years old woman who successfully underwent cardiac surgery with lead extraction and RV repair. With population aging, the incidence of complication after invasive procedures will grow. Leading with such complications in the elderly is challenging decision.

\section{Funding}

The Silvestre Adventist Network was responsible for payment of publishing costs.

\section{Conflict of interest}

None declared.

\section{Consent}

The author confirms that written consent for submission and publication of this case report including image(s) and associated text has been obtained from the patient.

\section{References}

[1] Hirschl D. A., Jain V. R., Spindola-Franco H. U., Gross J. N., and Haramati L B. 2007. "Prevalence and Characterization of Asymptomatic Pacemaker and ICD Lead Perforation on CT." Pacing and Clinical Electrophysiology 30 (1): 28-32.

[2] Werner, A. C. 2011. "The Older Population 2010." Accessed $\quad$ February 2018. https://www.census.gov/library/publications/2011/dec/c2 010br-09.html.

[3] Stevenson, R. T., Lugg, D., Gray, R., Hollis, D., Stoner, M., and Williams, J. L. 2012. "Pacemaker Implantation in the Extreme Elderly." J. Interv. Card. Electrophysiol. 33 (1): $51-8$.

[4] Banaszewski, M., and Stepinska, J. 2012. "Right Heart Perforation by Pacemaker Leads." Archives of Medical Science 8 (1): 11-3

[5] Nichols, J., Berger, N., Joseph, P., and Datta, D. 2015. "Subacute Right Ventricular Perforation by Pacemaker Lead Presenting with Left Hemothorax and Shock." Case Rep. Cardiol 2015: 983930.

[6] Schwerg, M., Stockburger, M., Schulze, C., Bondke, H., Poller, W. C., Lembcke, A., and Melzer, C. 2014. "Clinical, Anatomical, and Technical Risk Factors for Postoperative Pacemaker or Defibrillator Lead Perforation with Particular Focus on Myocardial Thickness." Pacing Clin Electrophysiol 37 (10): 1291-6.

[7] Laborderie, J., Barandon, L., Ploux, S., Deplagne, A., Mokrani, B., Reuter, S., et al. 2008. "Management of Subacute and Delayed Right Ventricular Perforation with a Pacing or an Implantable Cardioverter-Defibrillator Lead." Am. J. Cardiol. 102: 1352-5.

[8] Migliore, F., Zorzi, A., Bertaglia, E., Leoni, L., Siciliano, M., De, L. M., et al. 2014. "Incidence, Management, and Prevention of Right Ventricular Perforation by Pacemaker and Implantable Cardioverter Defibrillator Leads." Pacing Clin. Electrophysiol 37: 1602-9. 